\title{
Conocimiento y uso de las tecnologías de la información y de la comunicación en la formación médica continuada
}

\author{
Luis Rodríguez-Padial, María Luz Cacheiro-González, Antonio Medina-Rivilla
}

Introducción. Las tecnologías de la información y de la comunicación (TIC) han contribuido al desarrollo científico y social y deben desempeñar un papel esencial en la docencia y en la formación médica continuada (FMC). Aunque se han utilizado las TIC, presentan una serie de limitaciones que deben conocerse y corregirse para conseguir una mayor implantación de éstas. Por ello, se pretende conocer la experiencia y opinión que los médicos tienen sobre el uso de las TIC en la FMC.

Sujetos y métodos. Se ha utilizado un cuestionario por Internet alojado en la web de la Fundación Signo y se ha remitido un correo electrónico para recabar información de los médicos sobre las TIC. Se realiza análisis estadístico de los resultados.

Resultados. Se ha obtenido información de 661 profesionales del área de la salud, el 56,7\% de los cuales son varones. La edad media es de 48,9 años. El 30,6\% es de la provincia de Toledo y el 60\% realiza labor asistencial. La mayoría (76\%) tiene experiencia en la FMC, como alumnos (54\%) y como de docentes (19\%). La inclusión de materiales multimedia $(86,1 \%)$, la buena organización de las actividades $(76,1 \%)$ y la facilidad de uso $(74 \%)$ son los aspectos mejor valorados. La necesidad de constancia por parte del alumno (59\%), el idioma (39,6\%), la dificultad para evaluar el aprovechamiento $(35,5 \%)$ y para la interacción adecuada entre alumno y profesor $(23,6 \%)$ son las mayores dificultades. Se observa un escaso conocimiento de herramientas que podrían ser útiles, como Twitter y Facebook.

Conclusiones. Este colectivo tiene experiencia en el uso de las TIC en la FMC y valora claras ventajas, aunque señala inconvenientes que deben mejorarse para alcanzar un mayor uso de dichas tecnologías en la FMC.

Palabras clave. Docencia. Formación médica continuada. Tecnologías de la información y la comunicación.

\section{Knowledge and use of IT in medical lifelong learning}

Introduction. Information technologies (IT) have contributed to scientific and social development and play an essential role in teaching and in continuing medical education (CME). Although IT have been used, they have a number of limitations that should be known and corrected to improve implementation. The aim of this study was to know the degree of knowledge and application of IT in CME, and the advantages and disadvantages that doctors see on them.

Subjects and methods. A questionnaire hosted on the Internet website of the Signo Foundation was used and an email was sent to request information from doctors about IT in CME. Statistical analysis of the results was performed.

Results. Information was obtained from 661 individuals, $56.7 \%$ of whom were male. The average age was 48.9 years, $30.6 \%$ was living in the province of Toledo and $60 \%$ were on clinical work. Most (76\%) have experience in CME, both as students (54\%) and as teachers (19\%). The inclusion of multimedia materials (86.1\%), good organization of the course (76.1\%) and usability (74\%) are the most valued aspects of IT. The need of student effort and consistency (59\%), language other than Spanish (39.6\%), the difficulty in assessing learning (35.5\%) and limited interaction between students and teachers (23.6\%) were the greatest difficulties reported. Twitter and Facebook were not frequently used.

Conclusions. This experienced group in the use of IT in CME acknowledges clear advantages of these techniques, but also pointed out some disadvantages which should be solved to improve implementation.

Key words. Continuous medical education. Information technologies. Teaching.
Servicio de Cardiología; Complejo Hospitalario de Toledo (L. RodríguezPadial). Departamento de Didáctica, Organización Escolar y Didácticas Especiales; Facultad de Educación; Universidad Nacional de Educación a Distancia; Madrid, España (M.L. Cacheiro-González, A. Medina-Rivilla).

Correspondencia:

Dr. Luis Rodríguez Padial. Servicio de Cardiología. Complejo Hospitalario de Toledo. Avda. Barber, 30.

E-45004 Toledo.

E-mail:

Irpadial@gmail.com

Agradecimientos:

Al llustre Colegio Oficial de Médicos de Toledo y a la Fundación Signo, por su colaboración en la realización de la encuesta. Al Dr. Francisco Martín Rodríguez, por su apoyo en el análisis estadístico.

Conflicto de intereses: No declarado.

Competing interests: None declared.

(c) 2015 FEM 


\section{Introducción}

El impacto de las tecnologías de la información y la comunicación (TIC) en la sociedad ha sido enorme en los últimos años, habiendo contribuido notablemente a transformar tanto la educación [1] como la medicina. En medicina, las TIC desempeñan un papel muy importante en numerosos aspectos, que van desde la educación sanitaria a la gestión y seguimiento de pacientes crónicos, donde pueden mejorar la eficiencia [2]. El impacto de las TIC en medicina no se circunscribe a la atención sanitaria, sino que afecta de forma notable a la formación médica y también de manera importante a la formación médica continuada (FMC) [3,4], tema sobre el cual se ha tratado poco [5]. En este campo, el uso de las TIC se ve condicionado por el conocimiento y uso que los médicos tienen de ellas, que suele ser limitado [6-10].

La FMC es tanto un derecho como una obligación ética para el médico, especialmente relevante en un momento como el actual en el que existe un cambio tan continuado de todas las ramas de la medicina, al mismo tiempo que los profesionales disponen cada vez de menos tiempo para su formación. Por ello, es necesario el desarrollo de nuevas estrategias que permitan obtener la mejor actualización con la mayor eficiencia en tiempo y medios.

En un trabajo anterior, se ha estudiado el grado de conocimiento del uso de las TIC para el aprendizaje de la electrocardiografía [11] y se ha podido comprobar la actitud de los médicos sobre el uso de estas técnicas y la necesidad de establecer estrategias de mejora. No obstante, esta información se limita a una muestra muy específica y un campo de la formación muy concreto, por lo que parece razonable profundizar en el conocimiento sobre estas técnicas en una muestra mayor y más diversa, de manera que esta información pueda servir de base para establecer una estrategia dirigida a conseguir un mayor desarrollo de las TIC en el ámbito de la FMC.

Por ello, el objetivo de este trabajo ha sido conocer el grado de conocimiento que los médicos tienen de las TIC en la FMC y su uso en los procesos de FMC mediante una encuesta realizada por Internet.

\section{Sujetos y métodos}

\section{Población}

Se ha realizado una encuesta a través de Internet que ha estado alojada en la página web de la Funda- ción Signo (http://www.fundacionsigno.com), dedicada a mejorar la gestión y la evaluación de costes sanitarios. Se ha remitido un correo electrónico a todos los miembros y seguidores de dicha fundación, y a los médicos inscritos en el Colegio de Médicos de Toledo, pidiéndoles que contestaran dicha encuesta.

\section{Encuesta}

A través de Google Drive [12] se ha realizado una encuesta de 24 preguntas -que consta de preguntas cerradas de escalas de medición de actitudes y una respuesta abierta-, utilizando la escala de valoración de Likert con cinco respuestas. El cuestionario consta de varias partes: datos sociodemográficos y experiencia docente (cinco preguntas), experiencia en formación para médicos a través de Internet (cinco preguntas), experiencia en el uso de las TIC e Internet (tres preguntas), experiencia en uso de TIC en formación (nueve preguntas) y propuestas de mejora de la docencia médica a través de las TIC (dos preguntas). Se ha preguntado por las TIC consideradas por Cabero y Román [13]. En este trabajo se comentan las encuestas relacionadas con el conocimiento y uso de las TIC.

Para analizar la validez del cuestionario se realizó una evaluación por pares y un análisis de fiabilidad según el método de consistencia interna $\alpha$ de Cronbach. Se remitió a un grupo de expertos en metodología de la investigación y docencia y en medicina, al objeto de obtener sugerencias sobre el mismo. Se valoró la idoneidad de las preguntas, la fluidez del cuestionario y su capacidad de discriminación, entre otros aspectos del diseño. Las correspondientes sugerencias y comentarios fueron introducidos en el cuestionario definitivo. De nuevo, antes de remitir el cuestionario al total de los médicos encuestados, se realizó un estudio piloto, que se envió a un grupo aleatorio de 35 encuestados seleccionados entre el grupo total de estudio, al objeto de realizar el análisis de consistencia interna según el $\alpha$ de Cronbach, utilizando el programa estadístico Stata 12.

De estos registros se han recogido 26 respuestas, que se han incluido en al análisis de fiabilidad. Con estos cuestionarios se ha obtenido un $\alpha$ de Cronbach de 0,89, considerada adecuada al ser mayor de 0,7, el límite considerado habitualmente como indicativo de adecuada fiabilidad. El $\alpha$ de Cronbach obtenida en cada uno de los módulos ha sido superior a 0,75 (entre 0,77 y 0,9 ). Tras comprobar la consistencia interna del cuestionario, se ha procedido a enviarlo todos los médicos colegiados. 


\section{Análisis estadístico}

Los datos cualitativos se han expresado como proporción, y los cuantitativos, como media \pm desviación estándar o mediana más rango. Se han analizado las diferencias entre proporciones mediante la chi al cuadrado.

Se han considerado las tendencias en las respuestas según los grupos de edad (<30, 35-44, 45-59 y $\geq 60$ años), sexo, profesionales de Toledo y profesionales de la docencia, analizando en cada caso la diferencia con el grupo complementario. En el análisis de los datos por distintos estratos, se ha estudiado el número de respuestas y se han cuantificado las respuestas cualitativas según el siguiente código: 'experto', 5; 'alta', 4; 'regular', 3; 'baja', 2; 'nula', 1; 'NS/NC', missing. Esta 'transformación cuantitativa' de los datos ha permitido calcular la media de cada estrato de sexo y edad y obtener, con ello, una idea aproximada de la tendencia en la selección media de las respuestas.

Para el análisis estadístico se ha utilizado el programa Stata.12. En las comparaciones de dos colas se ha considerado significativo un valor de $p<0,05$.

\section{Resultados}

\section{Datos demográficos}

La encuesta ha sido completada por 661 profesionales, de los cuales 365 (56,7\%) son varones. La edad media ha sido de 48,9 años. De los encuestados, 56 tienen una edad inferior a 35 años. El 30,6\% $(n=202)$ es de la provincia de Toledo, mientras que la procedencia del resto es variada (21\% de Castilla y León, $10 \%$ de Madrid, $8 \%$ de Murcia, 4\% de Cataluña, por ejemplo).

\section{Experiencia docente y laboral}

Un total de 345 (52,2\%) participantes de los que contestaron a la encuesta reconocen ser docentes, aunque también es alto el número de encuestados que no lo son y sí tienen experiencia o interés por el uso de las TIC como usuarios $(n=316 ; 47,8 \%)$. La experiencia en la docencia es amplia, aunque presenta claras variaciones: la mayoría de los que han respondido a esta pregunta tienen una experiencia docente mayor de $5(n=254 ; 78 \%)$ o 10 años $(n=164$; $50 \%)$.

La mayoría (61\%) de los médicos que han respondido realizan una labor asistencial, aunque no es despreciable el número de los que están en pues- tos directivos (16\%) y en labores no asistenciales (10\%). Aunque la mayoría trabaja en hospitales (50\%), hay también un número significativo que lo hace en atención primaria (34\%).

\section{Experiencia en el uso de las TIC}

Un 76\% de los encuestados tienen experiencia en la formación a distancia de médicos y un $75 \%$ reconoce haber utilizado personalmente sistemas de e-learning. Los individuos que han respondido a la encuesta tienen amplia experiencia como alumnos de sistemas de formación a distancia $(n=358 ; 54 \%)$, aunque es significativo el número de los que reconocen haber trabajado también como docentes ( $n=123 ; 19 \%)$.

Al evaluar su experiencia en el uso de las distintas tecnologías (Tabla I), un alto porcentaje se considera con experiencia alta $(47,8 \%)$ y, en menor medida, regular (27\%). Es de destacar que el porcentaje de encuestados que se considera experto en el uso de estas tecnologías es bajo (7,5\%). La experiencia alta ocurre con mayor frecuencia en el uso de los ordenadores $(68,2 \%)$ que en el tratamiento de imágenes $(28,8 \%)$ o en el empleo de hojas de cálculo (33\%), siendo intermedia en el resto (42,8-57\%). En cuanto a los dispositivos, la frecuencia de una experiencia de nivel alto o de experto es elevada en aquellos más utilizados en la formación, como teléfono móvil (62,8\%), tabletas digitales $(49,4 \%)$ o teléfonos móviles inteligentes $(58,2 \%)$. Con respecto a la experiencia en el uso de programas, el procesador de textos $(63,6 \%)$ y la presentación con diapositivas $(70,9 \%)$ son los más conocidos por los encuestados.

El número de los que reconocen tener un nivel de experto o alto en el uso del ordenador es significativamente mayor del que reconoce tenerlo en el teléfono móvil $(p<0,001)$, de igual forma que quienes reconocen tener un nivel experto-alto en el uso de hojas de cálculo son significativamente menos numerosos que los que tienen dicha capacidad en el uso de procesadores de textos $(p<0,001)$, pero más frecuentes que los que reconocen ese nivel en el uso de sistemas para tratar imágenes $(p=0,0096)$.

Con respecto a la experiencia en el uso de distintas herramientas de Internet (Tabla II), la mayoría considera que tiene una experiencia alta en Internet $(78,4 \%)$ y navegadores $(62,2 \%)$, pero regular o baja en el resto de las herramientas consideradas. Destaca el hecho de que la mayoría de los participantes reconoce tener una experiencia baja o nula en el uso de las redes sociales (Facebook, Twenti y Twitter), dado que un nivel experto-alto en estas redes es reconocido por sólo el 3,6-13\%. El número 
Tabla I. Experiencia de uso de distintos dispositivos y programas $(n=661)$.

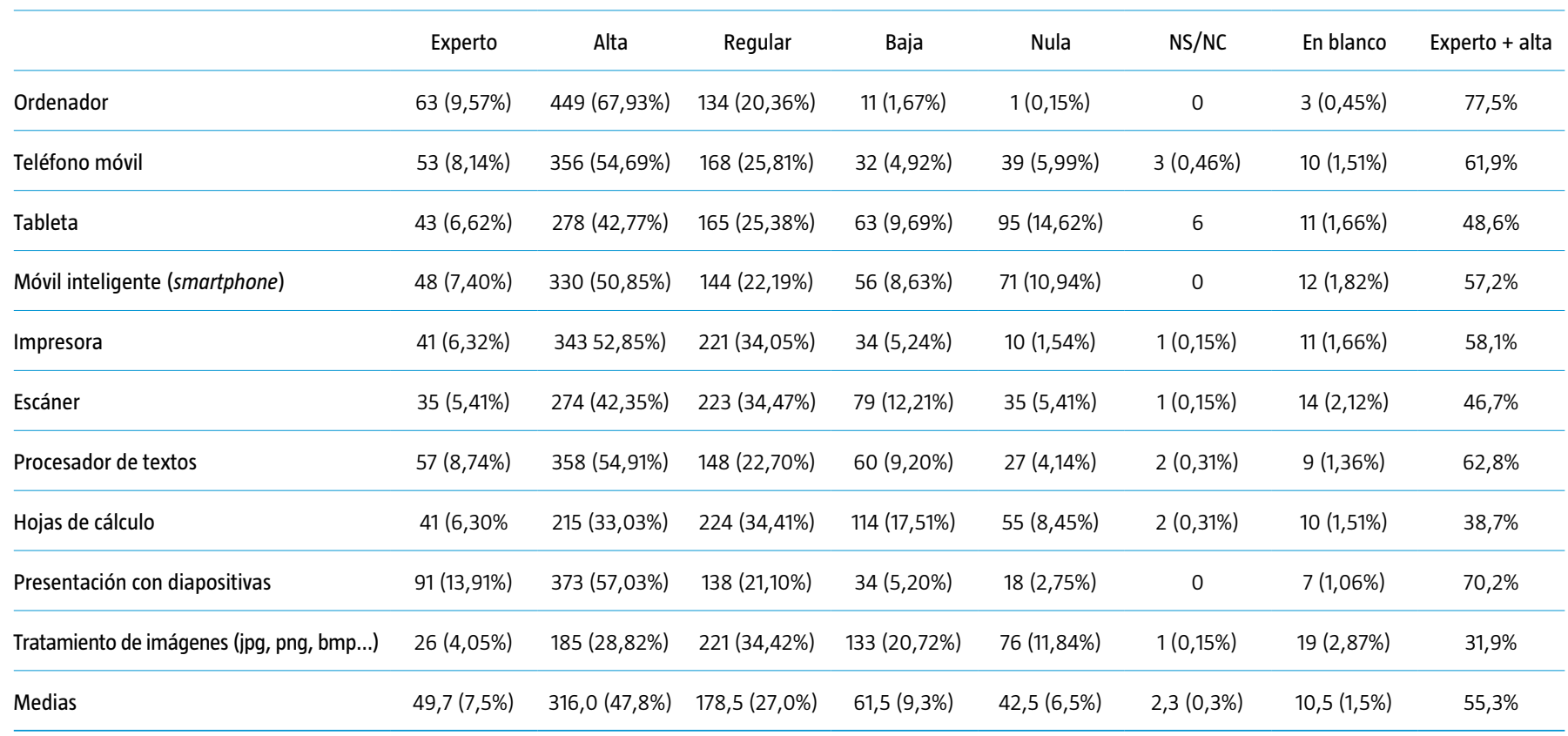

de quienes dicen utilizar Internet con un nivel experto-alto es significativamente mayor de los que se reconocen el mismo nivel en el uso de navegadores $(p<0,001)$, de igual forma que éstos son significativamente más numerosos que los que dicen usar los chats con este mismo nivel $(p<0,001)$. Twitter es utilizado con un nivel experto-alto por un número de sujetos significativamente menor que los blogs $(p=0,0263)$ o Facebook $(p<0,001)$.

\section{Patrón de uso de las TIC}

Con respecto al uso que realizan de las TIC, la mayoría indica que las utiliza para cursos presenciales $(31,8 \%)$, aunque es importante el número que las usan para consultar información $(24,1 \%)$ o artículos científicos a través de Internet (13,8\%). Por el contrario, el número de los que las utilizan para impartir cursos con plataformas educativas es bastante menor $(7,7 \%)$, al igual que ocurre con los que usan las TIC para compartir archivos (2,4\%). El número de los que utilizan las TIC para compartir información con compañeros médicos (3,63\%), asesorar alumnos $(2,1 \%)$ o participar en foros $(1,2 \%)$ es escaso, aunque no tanto como el de los que las utilizan para chatear con alumnos $(0,2 \%)$ o profesores $(0,45 \%)$. El número de los que las utilizan para cursos presenciales es significativamente mayor del que las usan para consultar información $(p=0,0018)$, de igual forma que éstos son significativamente más numerosos que los que consultan artículos científicos por Internet $(p<0,001)$.

\section{Opinión sobre las TIC}

La mayoría piensa que la página web $(75,3 \%)$ es el recurso TIC que puede ser más útil en la docencia, mientras que una minoría cree que el resto de las herramientas puede desempeñar un cierto papel. Entre otros recursos que pueden ser de interés para la docencia, destaca el blog (10,3\%). El resto de los recursos (wiki, Twitter y otros) son considerados de utilidad por una minoría $(2,1-4,7 \%)$ de los encuestados. El número de los que utilizan Twitter o alguna wiki es significativamente menor que el de los que usan algún blog $(p<0,001)$.

Cuando son preguntados sobre las ventajas de las TIC en la docencia, los encuestados consideran que en la mayoría de los casos las TIC tienen muchas o bastantes ventajas en la organización y gestión de los cursos. La posibilidad de incluir materiales multimedia $(86,1 \%)$, la utilidad en la organización del curso $(76,1 \%)$ y la facilidad de uso de la plataforma (74\%) constituyen los aspectos mejor valorados por los encuestados, aunque es de destacar que todos los aspectos evaluados sobre las TIC 
Tabla II. Experiencia de uso de distintas herramientas de Internet $(n=661)$.

\begin{tabular}{|c|c|c|c|c|c|c|c|}
\hline & Experto & Alta & Regular & Baja & Nula & $\mathrm{NS} / \mathrm{NC}$ & Experto + alta \\
\hline Internet & $57(8,62 \%)$ & $461(69,74 \%)$ & $133(20,12 \%)$ & $7(1,06 \%)$ & $2(0,30 \%)$ & $1(0,15 \%)$ & $78,4 \%$ \\
\hline Robots de búsqueda & 31 (4,69\%) & $184(27,84 \%)$ & $209(31,62 \%)$ & $119(18,00 \%)$ & $107(16,19 \%)$ & 11 (1,66\%) & $32,5 \%$ \\
\hline Foros de discusión & $23(3,48 \%)$ & $139(21,03 \%)$ & $244(36,91 \%)$ & $177(26,78 \%)$ & $77(11,65 \%)$ & $1(0,15 \%)$ & $24,5 \%$ \\
\hline Facebook & $29(4,39 \%)$ & $123(18,61 \%)$ & $146(22,09 \%)$ & $160(24,21 \%)$ & $199(30,11 \%)$ & $4(0,61 \%)$ & $23,0 \%$ \\
\hline Twitter & $22(3,33 \%)$ & $64(9,68 \%)$ & $92(13,92 \%)$ & $159(24,05 \%)$ & $319(48,26 \%)$ & $5(0,76 \%)$ & $13,0 \%$ \\
\hline Wikis & $13(1,97 \%)$ & $59(8,93 \%)$ & $123(18,61 \%)$ & $143(21,63 \%)$ & $313(47,35 \%)$ & $10(1,51 \%)$ & $10,9 \%$ \\
\hline YouTube & $24(3,63 \%)$ & $167(25,26 \%)$ & $221(33,43 \%)$ & $158(23,90 \%)$ & $89(13,46 \%)$ & $2(0,30 \%)$ & $28,9 \%$ \\
\hline Google Docs & $23(3,48 \%)$ & $152(23,00 \%)$ & $174(26,32 \%)$ & $147(22,24 \%)$ & $157(23,75 \%)$ & $8(1,21 \%)$ & $26,5 \%$ \\
\hline Plataformas educativas & $25(3,78 \%)$ & $167(25,26 \%)$ & $202(30,56 \%)$ & $150(22,69 \%)$ & $110(16,64 \%)$ & $7(1,06 \%)$ & $29,0 \%$ \\
\hline Skype & $22(3,33 \%)$ & $136(20,57 \%)$ & $189(28,59)$ & $140(21,18 \%)$ & $169(25,57 \%)$ & $5(0,76 \%)$ & $23,9 \%$ \\
\hline Windows Live & $13(1,97 \%)$ & $82(12,41 \%)$ & $137(20,73 \%)$ & $168(25,42 \%)$ & $251(37,97 \%)$ & $10(1,51 \%)$ & $14,4 \%$ \\
\hline Slideshare & $13(1,97 \%)$ & $71(10,74 \%)$ & $126(19,06)$ & $153(23,15 \%)$ & $285(43,12 \%)$ & $13(1,97 \%)$ & $12,7 \%$ \\
\hline
\end{tabular}

como ventajas han sido valorados positivamente por los participantes, lo que indican su buena percepción de la utilidad de las TIC en la docencia (Tabla III).

Al ser interrogados sobre las dificultades más importantes de los métodos de docencia médica a través de Internet, los encuestados consideran que la necesidad de constancia por parte del alumno (59\%) es la mayor de estas dificultades, seguida por la dificultad del idioma, dado que muchos cursos de calidad están en un idioma distinto al castellano (39,6\%). La dificultad para evaluar adecuadamente el aprovechamiento del alumno $(35,5 \%)$ y para realizar una interacción adecuada entre alumno y profesor $(23,6 \%)$ son otras de las dificultades seleccionadas por los encuestados.

\section{Diferencias por edad y sexo}

Al hablar sobre la experiencia en el uso de las distintas TIC, las mujeres tienden a puntuarse de forma similar a los hombres, aunque con algunas variaciones según la TIC considerada (puntuación total de 34,2 frente a 34 , con puntuaciones medias de 3,4 y 3,4 , respectivamente).

Los participantes han sido clasificados en distintos grupos etarios ( $<30,35-44,45-59$ y $\geq 60$ años) $y$ se ha observado que, al incrementarse la edad, la puntuación tiende a ser menor (puntuación total de 35,6 frente a 34,6, 34 y 33 para los grupos de edad considerados, con puntuaciones medias de 3,6, 3,5, 3,4 y 3,3 , respectivamente). Así, las personas de mayor edad tienden a considerar que tienen menor ex- 
periencia en el uso de las TIC con fines docentes. Estos resultados se mantienen de forma similar cuando se considera el sexo dentro de cada uno de los grupos de edad considerados. Con respecto a la experiencia sobre Internet y sus herramientas, las mujeres tienden a puntuarse por debajo de los hombres (puntuación total de 26,5 frente a 25,3, con puntuaciones medias de 2,6 y 2,5, respectivamente), excepto en Facebook y Tuenti.

De igual forma, a medida que aumenta la edad, la puntuación tiende a ser menor para todas las herramientas de Internet consideradas (puntuación total de 32,37 frente a 26,82, 25,44 y 22,96 para los grupos de edad considerados, con puntuaciones medias de $3,2,2,7,2,5$ y 2,3 , respectivamente). Estas puntuaciones son inferiores a las que los participantes se dan con respecto a las TIC no basadas en Internet. Las tendencias indicadas entre hombres y mujeres se mantienen cuando se consideran los sexos en cada uno de los grupos de edad considerados.

\section{Diferencias entre distintos grupos de encuestados}

No se observan diferencias entre los no docentes y los docentes al analizar el grado de conocimiento de las TIC, siendo la puntuación total alcanzada de 34,7 para los primeros y 33,6 para los segundos, con medias de puntuación de 3,5 y 3,4, respectivamente. De igual forma, tampoco se observan diferencias significativas en la puntuación que han asignado los docentes en comparación con los no docentes con respecto a las ventajas de las herramientas TIC en la docencia (puntuación total de 52,7 frente a 52,8; puntuaciones medias de 4,1 y 4,1 , respectivamente).

Al comparar los encuestados de Toledo con los procedentes de otras comunidades autónomas, tampoco se han observado diferencias significativas, aunque tienden a puntuarse con un valor ligeramente inferior en el conocimiento de las TIC (total de puntos de 34,1 frente a 34,4 , con puntuaciones medias de 3,4 y 3,4 , respectivamente). Cuando se consideran las distintas herramientas de Internet, los profesionales médicos de Castilla-La Mancha se califican con una puntuación ligeramente inferior que los de las otras comunidades autónomas (total de puntos de 25,2 frente a 26,4 , con puntuaciones medias de 2,5 y 2,6, respectivamente), sin que tampoco se observen diferencias con respecto a la evaluación de las ventajas de las TIC.

\section{Discusión}

En este trabajo se ha evidenciado que existe un gra- do de conocimiento y uso de las TIC muy variable en función de las técnicas consideradas, lo cual puede limitar la difusión de su uso en la FMC. También se observan diferencias menores en función del sexo y la edad de los individuos encuestados. Los datos obtenidos son especialmente relevantes en un momento en el que existe una explosión en el desarrollo de herramientas TIC [14], pero en el que se adolece de escasa información sobre la opinión de los profesionales que deben utilizarlas, especialmente en el terreno de la FMC [5].

Se ha estudiado el papel e impacto de algunas TIC en la docencia. En la Universidad de Edimburgo se han analizado datos acerca de las experiencias, conocimientos y actitudes ante las TIC de los nuevos estudiantes desde 1990 [15]. Posteriormente, el informe SEUSSIS evaluó el uso de las TIC en siete universidades europeas (Reino Unido, Finlandia, Noruega, Países Bajos, Italia, Francia y España), recogiendo datos de los años 2001-2002 [16], que se han obtenido especialmente en alumnos de medicina $[6,8,9,17]$ y profesionales de la salud [7], y han mostrado una situación muy similar a la descrita en nuestro trabajo, aunque con las diferencias propias de las distintas circunstancias. En nuestra muestra, la mayoría de los encuestados tiene experiencia en la FMC, y lo han hecho como estudiantes y profesores, lo que permite obtener una visión más amplia y realista sobre la utilidad de las TIC en nuestro medio.

La mayor experiencia en el uso de las TIC se debe a cursos completos, aunque también se dan los ejemplos de comentarios sobre casos clínicos e imágenes, así como la visualización y estudio de temas sueltos. En cuanto a las herramientas, el mayor conocimiento de las TIC se obtiene en el ordenador, procesador de textos y presentación de diapositivas. Las hojas de cálculo y las técnicas de gestión de imágenes son las TIC en las que los encuestados reconocen tener menor grado de conocimiento. Las mujeres y las personas de mayor edad refieren tener un menor grado de conocimiento de las TIC, lo que indica la existencia de cierta brecha digital entre edades y sexos.

Resultados similares han sido comunicados por otros autores en estudiantes de medicina [18], aunque los métodos de aprendizaje están cambiando en los últimos años, dado que se está imponiendo en las facultades de medicina el aprendizaje basado en la resolución de problemas, lo cual puede ser un excelente complemento a los métodos de docencia tradicionales en una formación científico-técnica como la que es necesaria en medicina [19]. Por el contrario, existe poca experiencia en la utilización de pro- 
Tabla III. Ventajas de las TIC en las actividades de FMC $(n=661)$.

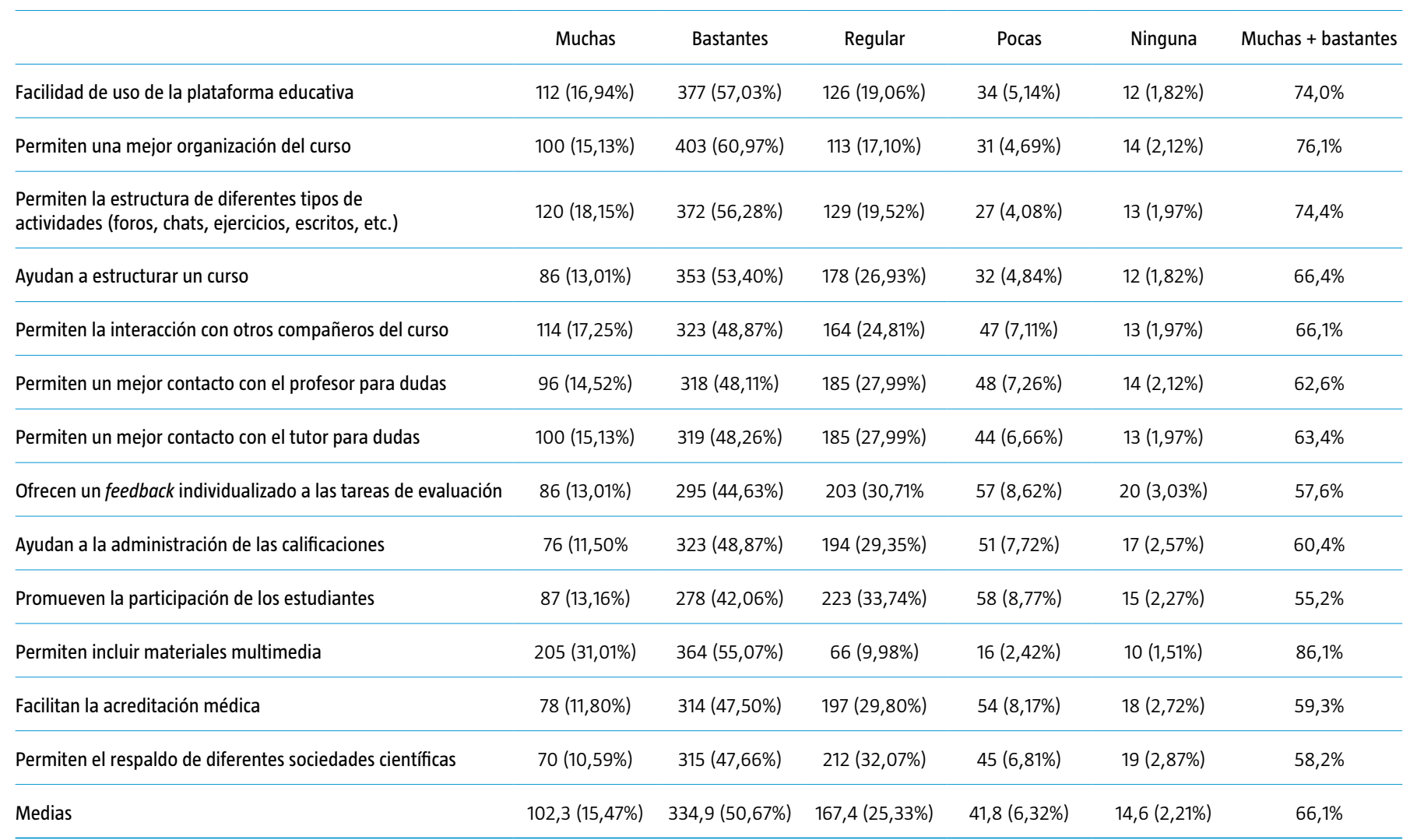

yectos colaborativos en la enseñanza de la medicina [20], aunque es probable que esta experiencia se incremente con la modificación de la enseñanza (plan Bolonia), que conlleva mayor actividad de colaboración en la enseñanza.

El teléfono móvil se ha convertido en el medio de comunicación más extendido en el mundo y tiene un gran potencial en FMC, el denominado mobile learning o aprendizaje móvil [21,22]. Para potenciar el uso de las TIC en la FMC, especialmente mediante la utilización de teléfonos y tabletas, se requiere un esfuerzo, sobre todo dirigido a las personas de mayor edad, lo que ayudaría a reducir la brecha digital. Diversos autores han estudiado el problema [23] y se han propuesto estrategias para mejorar la formación del personal sanitario en el uso de las TIC, con la intención de disminuir esta brecha digital [24] y facilitar el uso de las nuevas tecnologías, que en algunos casos han demostrado ser eficaces en la docencia [25], aunque también se ha observado que presentan limitaciones para la enseñanza de algunas disciplinas [26].
Las TIC pueden contribuir a desarrollar el hábito de trabajo en grupo porque hay determinadas estrategias metodológicas que promueven el trabajo en grupo y colaborativo [1]. El cambio que las TIC producen en el alumno (más activo) y el profesor (más facilitador e informador) será muy positivo en la adaptación de los médicos al uso de la información y a un trabajo de mayor colaboración, como de hecho se ha producido en la práctica de la medicina [27].

Aunque es cierto que globalmente los jóvenes tienen mayor habilidad con las TIC que los mayores [28], dado que son nativos digitales, existe una gran variabilidad en cada grupo de edad, de forma un número significativo de jóvenes no está adecuadamente entrenado en el uso de las TIC en la docencia. Por ello, conviene realizar una evaluación individual para establecer las acciones de formación necesarias en cada caso, pues muchas veces los jóvenes tienen más confianza que competencia [28]. Sería adecuado incluir aspectos de tecnología digital en el currículo de los alumnos, de manera que recibieran la ayuda 
de sus profesores en su conversión en auténticos profesionales digitales, lo cual les permitirá continuar su docencia profesional continuada con el uso de las TIC. En nuestro caso, los docentes tienen un conocimiento similar a los que no lo son, lo que indica la necesidad de un esfuerzo adicional en la formación de este grupo de profesionales.

Cuando consideramos la experiencia de los encuestados sobre el uso de Internet y todas las herramientas útiles para la formación en la investigación desarrollada, vemos que si bien se utiliza Internet por una mayoría $(78 \%)$, especialmente con el uso de navegadores (62\%), el resto de las herramientas de Internet se utilizan con escasa frecuencia. Conocemos por nuestro estudio previo [11] que el mayor uso de Internet que realizan los médicos se ciñe al correo electrónico. Esto indica que debería desarrollarse una intensa labor de formación en estas herramientas de Internet, útiles en la FMC, si queremos llevar a cabo una actividad que sea eficaz.

Llama la atención el escaso grado de conocimiento de los encuestados sobre las redes sociales y sobre las wikis, lo que indica que si se desea desarrollar alguna actividad de formación a través de ellas (Twitter, por ejemplo) [29], la labor de formación sobre su uso debería de ser prioritaria. La continua expansión de estas redes y su diversificación en búsqueda de distintos perfiles de usuarios están abocando a que se utilicen de manera creciente en el proceso de enseñanza-aprendizaje [30].

Los chats y los foros de discusión son conocidos y utilizados también por un número reducido de encuestados, lo que resulta relevante si tenemos en cuenta que serían las herramientas de mayor utilidad para mejorar la relación entre profesores y alumnos durante cualquier actividad de FMC, y cuyo déficit se ha señalado como una de las limitaciones más importantes. Algo similar ocurre con herramientas que permiten el intercambio de documentación, como Google Drive, que presentan también un grado de conocimiento escaso. Vemos así que se nota y se critica ese déficit de relación interpersonal en las actividades llevadas a cabo a través de Internet, pero al mismo tiempo se desconocen y usan poco las herramientas que, en la actualidad, permitirían mejorar ese aspecto.

Estos datos indican que estamos comenzando a utilizar las TIC de una forma algo extendida, pero lo hacemos aún sin aprovechar todo su potencial, bien por problemas de diseño o por otros relacionados con la mentalidad de uso, muy próxima todavía al empleo de información con poca interacción. Podemos decir, por tanto, que nuestra cultura del aprendizaje va retrasada con respecto al amplio po- tencial que pueden ofrecer hoy día las TIC, y aunque existen herramientas que podrían facilitar su adaptación a ellas, nuestro escaso conocimiento contribuye a limitarnos aún más. Será necesario un mayor desarrollo y adecuación de la tecnología, pero también es fundamental que conozcamos y utilicemos más aquella tecnología que ya tenemos a nuestra disposición para superar las limitaciones que vemos en las TIC en la actualidad cuando se aplican al campo de la FMC [31].

Este estudio tiene algunas limitaciones que conviene considerar. En primer lugar, existe sesgo en la selección de la muestra porque la encuesta se ha remitido sólo a médicos que utilizan el correo electrónico o Internet. De igual forma, la muestra no es aleatoria, a pesar de lo cual consideramos que el número de encuestas recibido es elevado y puede ser razonable utilizar esta información en las mejora de las TIC porque, de momento, éstos son los profesionales que las utilizan.

En conclusión, los datos del estudio indican una adopción algo desigual de las TIC y un escaso conocimiento y uso de las redes sociales con utilidad en la FMC, por lo que es elevar el conocimiento de estas TIC si se pretende mejorar su eficiencia docente en la FMC. Además, existen algunas diferencias entre distintos grupos (jóvenes, mujeres) que conviene considerar en el desarrollo de proyectos de mejora.

\section{Bibliografía}

1. Prendes-Espinosa MP. Internet aplicado a la educación: estrategias didácticas y metodológicas. In Cabero-Almenara J, coord. Nuevas tecnologías aplicadas a la educación. Madrid: McGraw-Hill; 2007. p. 205-22.

2. Gaikwad R, Warren J. The role of home-based information and communications technology interventions in chronic disease management: a systematic literature review. Health Informatics J 2009; 15: 122-46.

3. Chang G, Cook D, Maguire T, Skakun E, Yakimets WW, Warnock GL. Problem-based learning: its role in undergraduate surgical education. Can J Surg 1995; 38: 13-21.

4. Nandi PL, Chan JN, Chan CP, Chan P, Chan LP. Undergraduate medical education: comparison of problem-based learning and conventional teaching. Hong Kong Med J 2000; 6: 301-6.

5. Gaglani SM, Toplol EJ. iMedEd: the role of mobile health technologies in medical education. Acad Med 2014; Jun 2. [Epub ahead or print].

6. Dorup J. Experience and attitudes towards information technology among first-year medical students in Denmark: longitudinal questionnaire survey. J Med Internet Res 2004; 6: e10.

7. Jadad AR, Sigouin C, Cocking L, Booker L, Whelan T, Browman G. Internet use among physicians, nurse, and their patients. JAMA 2001; 286: 1451-2.

8. Gouveia-Oliveira A, Rodrigues T, De Melo FG. Computer education: attitudes and opinions of first-year medical students. Med Educ 1994; 28: 501-7.

9. Nurjahan MI, Lim TA, Foong A, Yeonf SW, Ware J. Computer literacy in medical students. Med Educ 2000; 34: 966. 
10. Bravo-Toledo R. Contra la resistencia, innovación. Cibermedicina 2011; $1: 3$.

11. Rodríguez-Padial L, Cacheiro ML. Grado de conocimiento y aplicación de las nuevas tecnologías de la información y de la comunicación en la docencia de la electrocardiografía por los médicos de la provincia de Toledo. FEM 2014; 17: 21-30.

12. Alarco JJ, Álvarez-Andrade EV. Google Docs. Una alternativa de encuesta online. Educ Med 2012; 1: 9-10.

13. Cabero-Almenara J, Román-Graván P, coords. E-actividades. Un referente básico para la formación en Internet. Sevilla: Eduforma; 2005.

14. Sánchez J. El futuro de la mHealth: oportunidades de desarrollo para mantener prestaciones en épocas de crisis. El Médico 2014; 1150: 69-73.

15. Asgari-Jirhandeh N, Haywood J. Computer awareness among medical students: a survey. Med Educ 1997; 31: 225-9.

16. SEUSISS. Survey of European Universities Skills in ICT of Students and Staff; 2003

17. Valcke M, De Wever B. Information and communication technologies in higher education: evidence-based practices in medical education. Med Teach 2006; 28: 40-8.

18. Gil MC, Arnedo M, Ribate MP, Puisac B, Lanuza J, Sáenz MA, et al. Uso de las tecnologías de la información y comunicación (TIC) en las licenciaturas de bioquímica y medicina de la Universidad de Zaragoza. IX Encuentro Virtual Educa Zaragoza; 2008.

19. González-López E, García-Lázaro I, Blanco-Alfonso A, OteroPuime A. Aprendizaje basado en la resolución de problemas: una experiencia práctica. Educ Med 2010; 13: 15-24.

20. Parra P, Matus O, Ortiz L, Torres G, Ibacaches A, Ramírez L, et al. Enseñanza y evaluación del profesionalismo médico a través de proyectos colaborativos. Rev Educ Cienc Salud 2010; 7: 29-34

21. Brazuelo, F. Nuevos recursos móviles. In Gallego D, Alonso-
García C, Cacheiro ML, coords. Educación, sociedad y tecnología. Madrid: Ramón Areces/UNED; 2011. p. 337-66.

22. Sandars JE, Frith GS. Mobile learning (m-learning). In Dent JA, Harden RM, eds. A practical guide for medical teaching. London: Churchill Livingstone Elsevier; 2013. p. 231-6.

23. Stevens RJ, Hamilton NM. Is there a digital generation gap for e-learning in plastic surgery? J Surg Educ 2012; 69: 344-9.

24. Reinbeck DM, Fitzsimons V. Bridging nursing's digital generation gap. Nurs Manage 2014; 45: 12-4.

25. Al-Hadithy N, Ghosh S. Smartphones and the plastic surgeon. J Plast Reconstr Aesthet Surg 2013; 66: e155-61.

26. Edlin JC, Deshpande RP. Caveats of smartphone applications for the cardiothoracic trainee. J Thorac Cardiovasc Surg 2013; 146: 1321-6.

27. Cabero-Almenara J, Gisbert-Cervera M. El papel del profesor y el alumno en los nuevos entornos tecnológicos de formación. In Cabero-Almenara J, coord. Nuevas tecnologías aplicadas a la educación. Madrid: McGraw-Hill; 2007. p. 261-77.

28. Ellaway RH, Tworek J. The net generation illusion: challenging conformance to social expectation. In Ferris SP, ed. Teaching and learning with the net generation: concepts and tools for reaching digital learners. Hershey: Information Science Reference/IGI Global; 2012. p. 324-39.

29. Grosseck G, Hololescu C. Teacher education in 140 characters -microblogging implications for continuous education, training, learning and personal development. Proc Soc Behav Sci 2011; 11: 160-4.

30. Fumero A. Somos redes; somos profesionales. Un breve recorrido por la realidad de las redes sociales profesionales. Cibermedicina 2011; 1: 4-7.

31. Tárraga R, Fernández MI, Pastor G, Colomer C. Descripción de una experiencia para el desarrollo de la competencia tecnológica de modo transversal en la formación inicial del profesorado. REDU Revista de Docencia Universitaria 2013; 11: 383-402. 